\title{
LA LLUERA II (SAN JUAN DE PRIORIO, OVIEDO, ASTURIAS, ESPAÑA): ESTUDIO INTEGRAL DE UN SANTUARIO COMPLEMENTARIO SOLUTRENSE
}

\author{
La Lluera II (San Juan de Priorio, Oviedo, Asturias, Spain): \\ integral study of a complementary solutrean sanctuary
}

\author{
José Adolfo Rodriguez Asensio ${ }^{1}$ y José Manuel Barrera Logares ${ }^{2}$
}

Recibido el 13 de marzo de 2013. Aceptado el 13 de septiembre de 2013

Resumen. Localizada a 54,96 m de distancia de su homónima, aguas arriba y en la misma margen derecha del río Nalón, la cueva de La Lluera II (San Juan de Priorio, Oviedo, Asturias, España), a pesar de sus dimensiones reducidas (que más bien nos obliga a definirla como angosto covacho), presenta unas características tanto geológicas como culturales y artísticas, estudiadas mediante una metodología específica basada en la gestión meticulosa de la información intra-site, gracias al empleo de las tecnologías de exploración láser, fotogrametría de alta resolución (HD) y restitución digital en tres dimensiones (3D), que son de gran importancia a la hora de explicar los primeros momentos del poblamiento solutrense en la cuenca media del río Nalón durante el Tardiglacial.

Palabras clave: cuenca media del Nalón, cueva de La Lluera II, análisis intra-site, arte parietal, Solutrense.

Abstract. Located at 54,96 $\mathrm{m}$ of distance from the homonymous cave, upstream and in the same right side of the Nalón river, La Lluera Il cave (San Juan de Priorio, Oviedo, Asturies, Spain), in spite of its reduced dimensions (which would rather force us to define it as a small narrow cave), presents its own geological, cultural and artistic features. These characteristics are entirely studied by means of a specific methodology which is based on the meticulous management of the intra-site information, thanks to the use of the scan laser technologies, high resolution photogrammetry (HD) and three-dimensional digital restitution (3D), which results of great importance for explaining the first appearance of the solutrean settlement in the Nalón middle basin during the Late Glacial.

Key words: Nalón middle basin, La Lluera Il cave, intra-site analysis, rock art, Solutrean.

\section{INTRODUCCIÓN}

La cueva de La Lluera II (San Juan de Priorio, Oviedo, Asturias, España) fue localizada en el verano de 1982 durante los trabajos de prospección sistemática de toda la zona colindante a La Lluera I (San Juan de Priorio, Oviedo, Asturias, España) con el propósito de comprobar la posible existencia, en las múltiples cuevas y oquedades que existen en ambas orillas de este sector del río Nalón, de otros restos arqueológicos o artísticos que vinieran a complementar los hasta aquel momento descubiertos en ésta (Fortea 1989, 1990; Rodríguez Asensio 1992). De este modo, los arqueólogos Enrique Arnau y Maximino Suárez, miembros del equipo de excavación de La Lluera I, descubrieron a 54,96 m de La Lluera I, aguas arriba y en la misma margen derecha del río Nalón (Fig. 1), en la base de un abrupto escarpe rocoso, un angosto conducto de apenas cuatro metros de fondo, poco más de un metro de anchura y unos $80 \mathrm{~cm}$ de altura desde

(') Área de Prehistoria. Departamento de Historia. Universidad de Oviedo. Calle Teniente Alfonso Martínez s/n. E-33011 Oviedo. adolfo@uniovi.es () Calle Albéniz 12, 2.E-33011. Oviedo. jmebarrera@gmail.com 


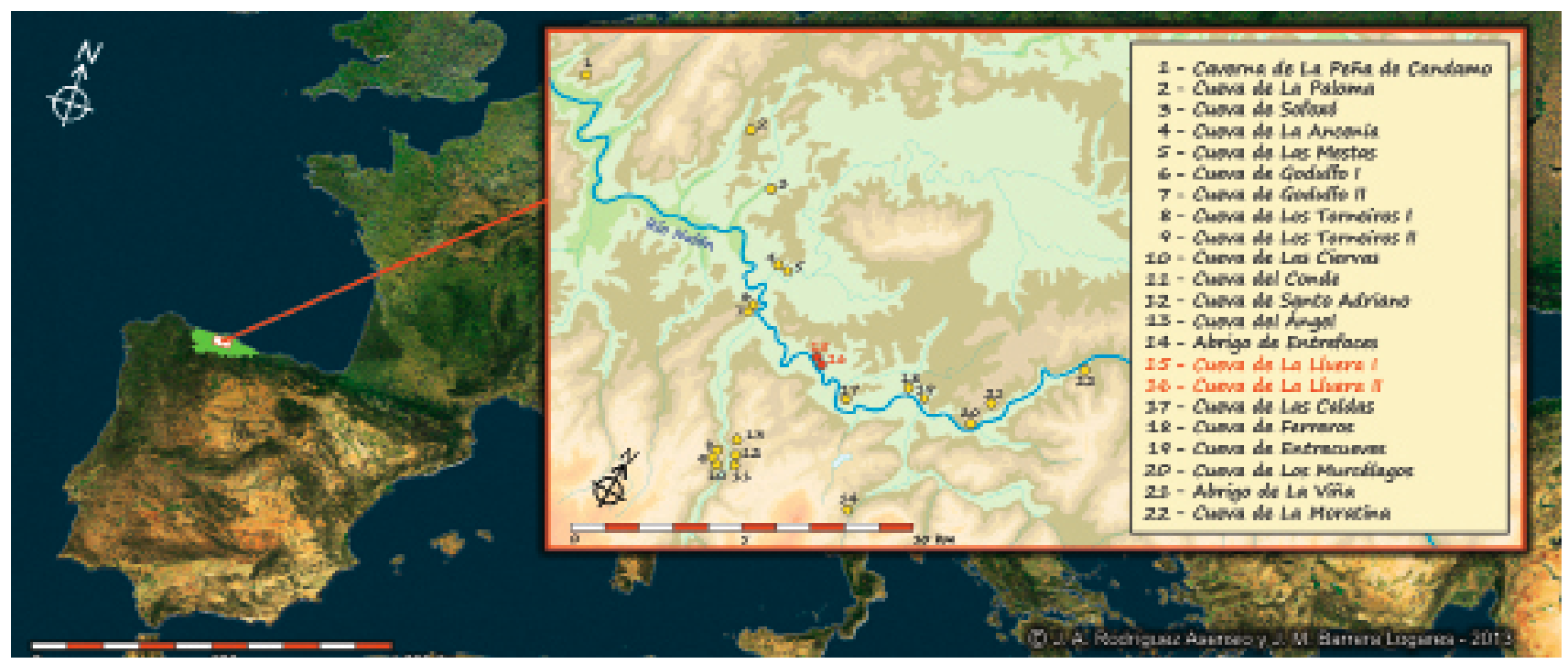

A Figura 1. Localización de las cuevas y abrigos con arte y/o yacimiento paleolítico de la cuenca media del río Nalón (Asturias, España). En rojo se destacan las cuevas de La Lluera.

un suelo que asciende en empinado buzamiento hacia una entrada cenital que se abre en el borde superior del escarpe y que presentaba en una de sus paredes grabados similares a los documentados en La Lluera I. Esta pequeña cavidad, aunque conocida por los vecinos de San Juan de Priorio, carecía de uso ni denominación alguna por lo que se decidió denominarla Lluera II por su proximidad y relación con La Lluera I y con tal nombre fue dada a conocer a la literatura científica (Fig. 2).

La geomorfología de esta reducida cavidad viene marcada por las mismas caracteristicas que dieron origen a la formación de La Lluera I y, por extensión, al amplio conjunto de cuevas y oquedades que orlan las orillas de los ríos Nalón, Nora y otros cursos subsidiarios (González Fernández et al. 2006) del área central de Asturias, intensamente karstificada debido al substrato de calcitas carboniferas sobre el que se asientan y que dan lugar a numerosas galerías y sumideros por los que las aguas de filtración y escorrentía de las laderas circundantes se canalizan y desaguan a la red fluvial.

A esta fenomenología interna viene a unirse la progresiva mecánica de encajamiento de estos ríos con sus niveles de aterrazamiento, formación de pequeños cañones con escarpes que apenas sobrepasan los $10 \mathrm{~m}$ de altura y erosiones motivadas por los fenómenos de desgaste lateral a que se ven sometidas sus orillas por el espectacular desarrollo de los meandros en esta zona. En el caso que nos ocupa, el curso del río Nalón discurre describiendo un amplio arco, después de salir de una curva muy pronunciada, que corta en vertical el roquedo de la margen derecha desviándose, a partir de ese punto, la mecánica de desgaste lateral y por tanto la formación de escarpes hacia la orilla opuesta, concavidad de este tramo de meandro. La orilla derecha se conforma en este punto como un amplio tramo de terraza en cuya unión con la base de la ladera colindante se localizan, aunque en cotas ligeramente distintas sobre el cauce fluvial, las dos cuevas de La Lluera.

Será precisamente esa diferencia de cotas y la característica peculiar de la presencia de un pequeño resalte rocoso en la base de la entrada inferior de La Lluera II lo que marcará un proceso de deposición sedimentológica diferenciada para las dos cuevas. Mientras que La Lluera I, con una cota de $+5,75$ m sobre la terraza actual, continuó viéndose expuesta a los diferentes procesos de inundación y vaciado causados por el río Nalón (Rodríguez Asensio 1990; Hoyos 1994, 1995); La Lluera II, con una cota de $+7,34 \mathrm{~m}$, se vio libre de tales fenómenos y su registro sedimentario obedecerá casi exclusivamente a los aportes por escorrentía desde su entrada superior.

El yacimiento arqueológico, de apenas $2,20 \mathrm{~m}^{2}$, fue excavado durante una campaña en el año 1988 (Rodríguez Asensio 1992), toda vez que se habian concluido los trabajos en La Lluera I y se disponía para ella de una estratigrafía suficientemente precisa (Rodríguez Asensio 1990), con la finalidad de poder relacionar ambos yacimientos culturalmente en el caso de que se localizaran en La Lluera II restos de ocupación antrópica, ya que su correspondencia artística había quedado fuera de toda duda durante la investigación preliminar (Fortea 1989, 1990, 1994). Estos trabajos dejaron de manifiesto la presencia de una secuencia estratigráfica muy elemental formada por un nivel de restos de terraza fluvial a base, sobre el que se asienta un nivel antrópico y sobre el que se registra, ya cerrando la serie, un nivel de limos de escorrentía en pronunciado buzamiento hacia la entrada inferior de la cueva (Rodríguez Asensio 1992).

Para el estudio intra-site de la cueva de La Lluera II, hemos procedido a la aplicación de una metodología espe- 


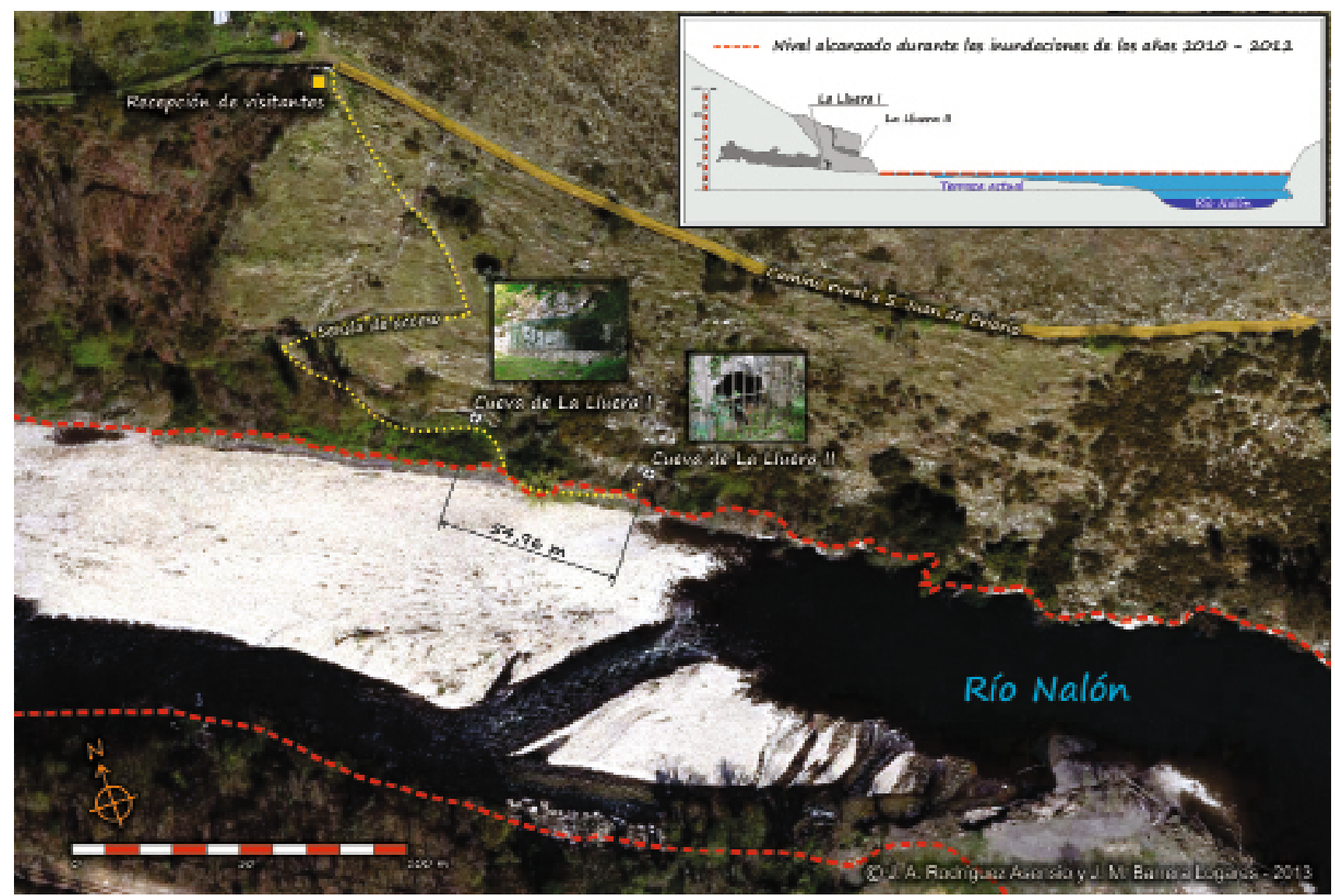

ム Figura 2. Vista aérea de la zona del río Nalón donde se localizan las cuevas de La Lluera. Se destaca con línea roja discontinua el nivel máximo alcanzado por el río durante los episodios de inundación que tuvieron lugar durante los años 2010 y 2012, que redundan en la noción de lo expuestas que están ambas cuevas, aún hoy en día, a los fenómenos producidos por la mecánica fluvial y que inciden inexorablemente en las posibilidades de habitación humana en ellas.

cifica basada en la gestión informática de los datos, tanto estratigráficos como arqueológicos, documentados con minuciosidad durante el proceso de excavación. A esto se sumó el análisis de la colección industrial, estableciéndose las comparativas tanto internas como las de concordancia con el registro lítico documentado en los diferentes niveles de La Lluera I y sus relaciones sedimentarias. Todo ello integrado por último mediante la modelización espacial tanto de las caracteristicas geomorfológicas y sedimentarias de la cueva como de las manifestaciones artísticas parietales presentes en ella y su registro arqueológico, gracias al empleo de tecnologías de escaneado laser y de fotogrametría de alta resolución (HD), restituyéndose digitalmente en tres dimensiones (3D) todo el conjunto de los datos.

\section{LA SERIE ESTRATIGRÁFICA}

El registro estratigráfico aporta, como ya hemos apuntado, un único momento de ocupación antrópica de unos 20 $\mathrm{cm}$ de potencia localizado sobre el conglomerado de cantos $y$ arenas limosas que conforman el estrato base de la se- cuencia sedimentológica de la cueva. Este depósito basal se encuentra directamente relacionado con una secuencia fluvial positiva de terraza de cota superior a los $+5 \mathrm{~m}$ (Hoyos 1994, 1995) e interpretado (Fig. 3) como una parada con escaso efecto de relleno en el proceso de incisión del valle iniciado por el río Nalón desde la terraza de $+8 \mathrm{~m}$ atribuida al último interglacial (Hoyos 1989). Secuencia que también se encuentra documentada en la base estratigráfica de La Lluera I.

Debido a la particular morfología de la base de la entrada de la cueva, constituida por un umbral de roca aflorante que actúa como muro de contención, los citados materiales fluviales así como el nivel de ocupación antrópica quedaron protegidos de las subsiguientes erosiones, tanto de las aguas del río Nalón como de las producidas por las aguas de escorrentía interior provenientes de la abertura cenital existente en el techo al fondo de la cueva. Dicha escorrentía, sin embargo, aportó los sedimentos limosos que contribuyeron a sellar el yacimiento posteriormente y colmataron la cueva hasta la actualidad (Fig. 4).

La consecuencia directa de este contexto sedimentológico, marcado inexorablemente en su inicio por las secuencias fluviales del río Nalón, es la imposible habita- 


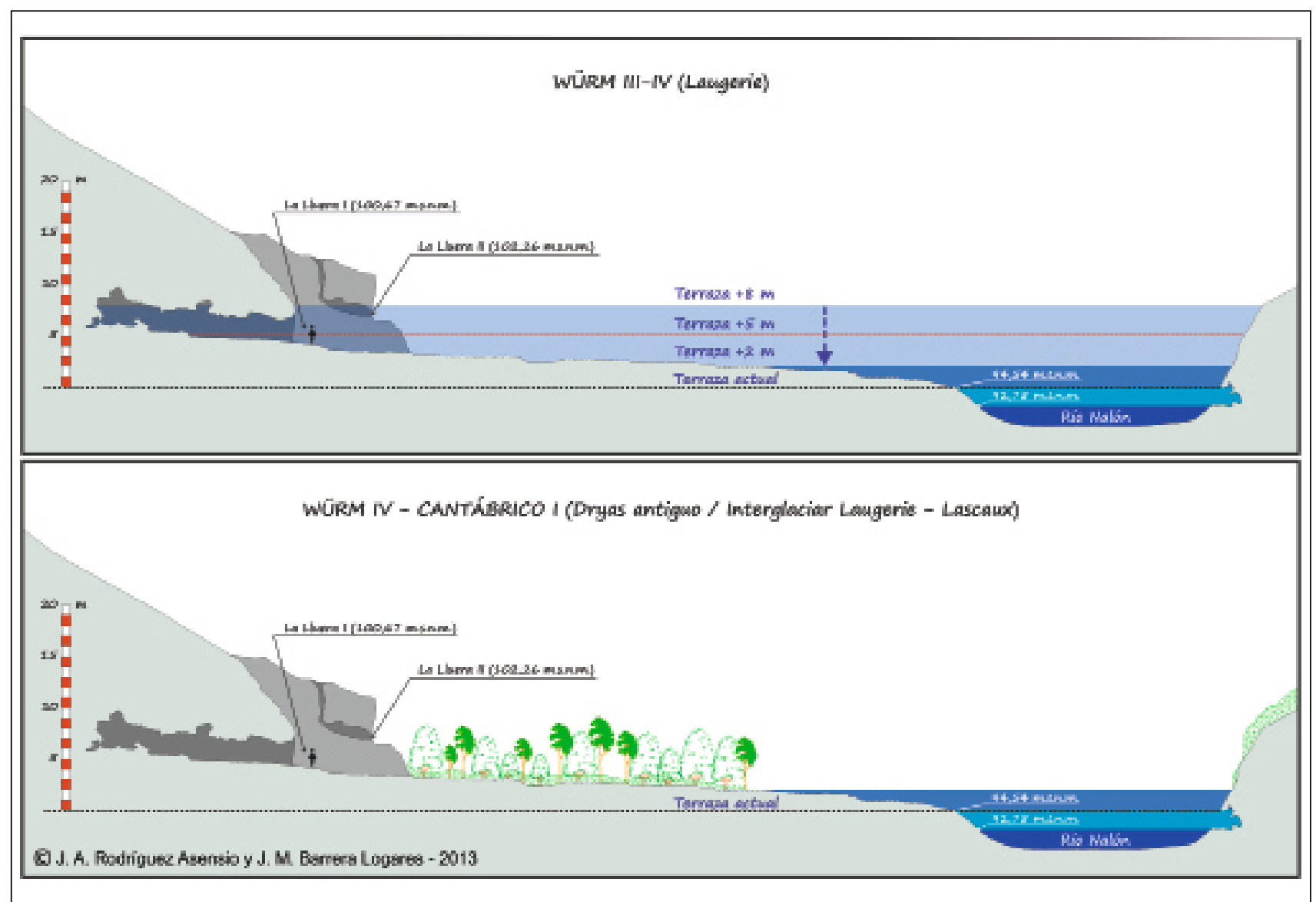

A FIgURA 3. Evolución de los niveles de terraza en la zona del río Nalón donde se encuentran las cuevas de La Lluera durante el Würm III-IV según M. Hoyos (1995). Será a partir del desmantelamiento de la terraza + $5 \mathrm{~m}$ cuando se produjo el vaciado de La Lluera I y, ya durante el Cantábrico I, se dieran las condiciones de no intrusión fluvial que posibilitaron su habitabilidad.

bilidad de la oquedad con anterioridad a la formación del estrato base, esto es del depósito de terraza de $+5 \mathrm{~m}$, lo que implica que tanto el registro industrial como las manifestaciones artísticas grabadas en la pared occidental de la cueva han de ubicarse cronológicamente en momentos posteriores a él.

Si además, tenemos en cuenta que ambas cuevas de La Lluera han de tomarse, desde el punto de vista del asentamiento humano, como un único conjunto indivisible y La Lluera I se encuentra en una cota más baja, el nivel de ocupación localizado en La Lluera II necesariamente habrá de ser, como muy temprano, simultaneo a las primeras ocupaciones detectadas en ésta (Rodríguez Asensio 1990). A saber, con posterioridad a la erosión postdeposicional de los depósitos de terraza de cota $+5 \mathrm{~m}$ generados durante las últimas pulsaciones de la fase climática Würm III-IV localizadas en la base estratigráfica de La Lluera I (Hoyos 1994, 1995). Esta afirmación también se ve corroborada por la dificultad orográfica que plantea su acceso, impracticable desde la entrada cenital, y que obliga tanto en la actualidad como en los tiempos prehistóricos a pasar primero por La Lluera I y a seguir la única senda transitable por la orilla del rio hasta su entrada inferior (Fortea 1990).

\section{EL REGISTRO INDUSTRIAL}

El estudio del registro material (Tab. 1) se llevó a cabo mediante el análisis de tipologías, cadenas operativas, y material soporte. La información aportada por cada una de las disciplinas mencionadas se integró a su vez dentro del análisis de conjunto del nivel fértil, determinado por la distribución espacial de los diferentes elementos del registro arqueológico (Fig. 5). Desde este punto de vista quedó establecido que los distintos elementos presentes no se encuentran directamente interrelacionados, ni conforman agrupaciones distintivas, de modo que las caracteristicas generales del conjunto no permiten hablar de que en el lugar se produjera el desarrollo de una actividad concreta.

Esta aparente anarquia espacial pudiera ser interpretada como debida a movimientos postdeposicionales del registro motivados por los arrastres producidos por las aguas de escorrentía provenientes de la entrada superior; sin embargo esta explicación no resulta plausible, pues de ser así y teniendo en cuenta el marcado buzamiento del sedimento de limos superior, los registros habrian de aparecer agrupados hacia la zona de desagüe, muy próximos o directamente agrupados en conexión con el resalte rocoso 


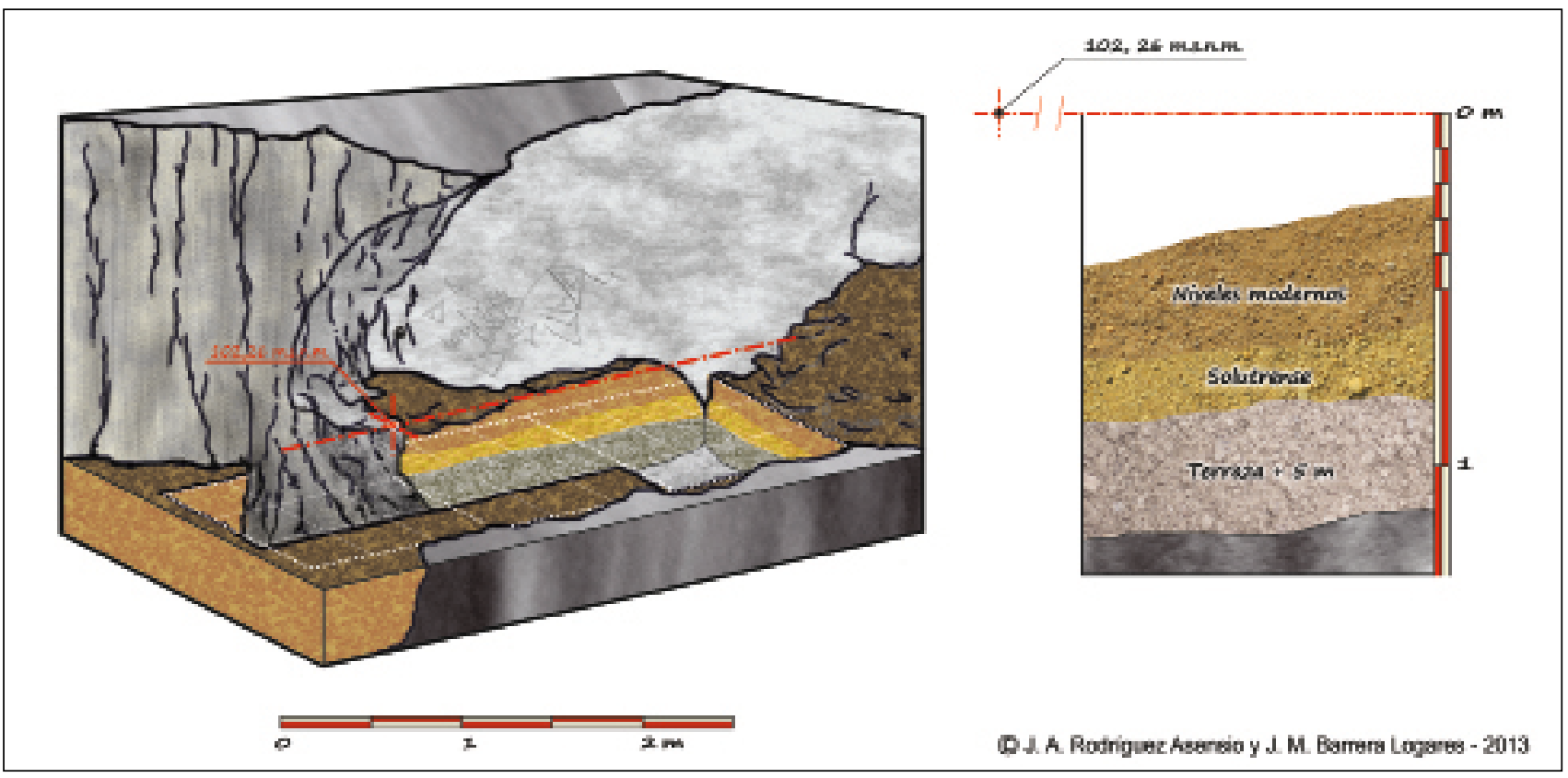

A FiguRA 4. Sección isonométrica y estratigrafía de la Lluera II.

\begin{tabular}{|c|c|c|c|c|}
\hline \multirow{2}{*}{\multicolumn{2}{|c|}{ Secuencia Paleoclimática }} & \multicolumn{3}{|c|}{ Cantábrico I (Dryas antiguo) } \\
\hline & & \multicolumn{3}{|c|}{ Fase 3} \\
\hline \multicolumn{2}{|r|}{ Secuencia Cultural } & \multicolumn{3}{|c|}{ Solutrense superior } \\
\hline \multicolumn{2}{|r|}{ Material } & Sílex & Cuarcita & Total \\
\hline \multirow{8}{*}{$\stackrel{ }{\stackrel{2}{E}}$} & Punta de Cara Plana & 1 & 4 & 5 \\
\hline & Pieza denticulada & 0 & 1 & 1 \\
\hline & Perforador-Raspador & 1 & 0 & 1 \\
\hline & Perforador & 1 & 0 & 1 \\
\hline & Pieza con retoques continuos en un borde & 2 & 1 & 3 \\
\hline & Raspador aquillado & 1 & 0 & 1 \\
\hline & Raedera & 0 & 2 & 2 \\
\hline & Raspador simple en extremo de hoja & 1 & 0 & 1 \\
\hline \multicolumn{2}{|r|}{ Total de registros } & 7 & 8 & 15 \\
\hline \multicolumn{2}{|r|}{$\%$ Total } & $46,67 \%$ & $53,33 \%$ & $100,00 \%$ \\
\hline \multicolumn{2}{|r|}{ Material } & Sílex & Cuarcita & Total \\
\hline \multirow{7}{*}{$\stackrel{\circ}{\stackrel{2}{=}}$} & Núcleo & 0 & 0 & 0 \\
\hline & Lasca simple & 8 & 7 & 15 \\
\hline & Lasca de decorticado primario & 1 & 2 & 3 \\
\hline & Lasca de decorticado secundario & 0 & 12 & 12 \\
\hline & Hoja & 1 & 7 & 8 \\
\hline & Hojita & 0 & 0 & 0 \\
\hline & Restos & 15 & 10 & 25 \\
\hline \multicolumn{2}{|r|}{ Total de registros } & 25 & 38 & 63 \\
\hline \multicolumn{2}{|r|}{ \% Total } & $39,68 \%$ & $60,32 \%$ & $100,00 \%$ \\
\hline
\end{tabular}

\ TABLA 1. Registro industrial por número de objetos documentados en la cueva de La Lluera II.

que delimita la entrada inferior y no como se documentaron, conformando un área de dispersión espacial, coincidente además con la zona de pared donde se ubica el panel de los grabados.
La ausencia absoluta de núcleos así como la escasa presencia de desechos de talla, se contabilizan en esta categoría 25 piezas, implica que en la cueva no se realizaron actividades propias de la talla o que éstas se vieron reducidas a su 


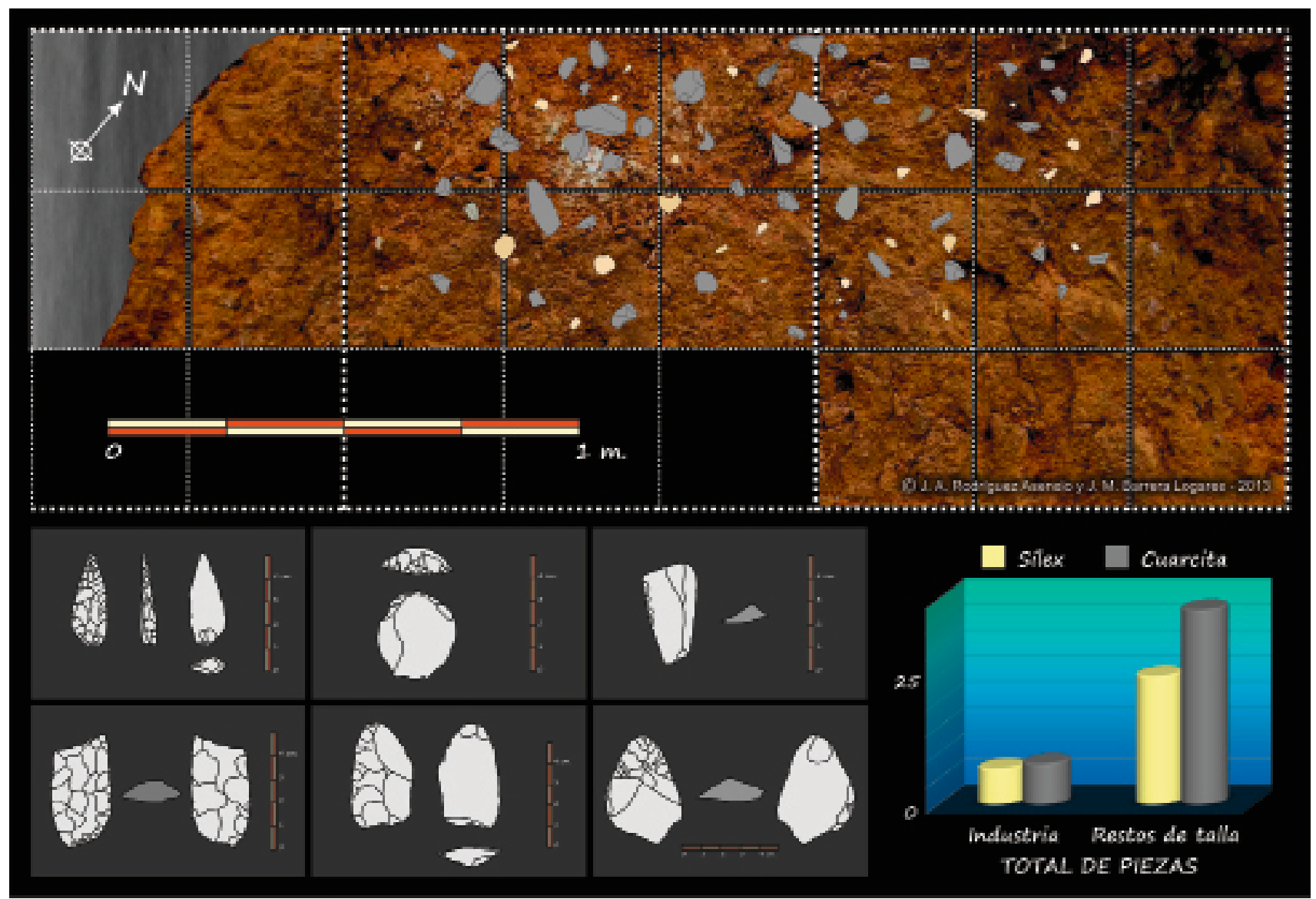

\ Figura 5. Planta del nivel fértil de La Lluera II con la disposición espacial de los hallazgos arqueológicos. En detalle, industria y comparativa del total de materiales documentados.

mínima expresión. Lo cual quedaría explicado a priori por las nulas condiciones de habitabilidad de esta angosta oquedad. Sin embargo, hemos de notar la calidad tanto técnica como de conservación de los escasos elementos industriales documentados, un conjunto de 15 piezas compuesto por tres raspadores, dos raederas, varios perforadores, piezas retocadas, etc. y en el que destacan cuatro puntas de cara plana en cuarcita y una en sílex con retoque cubriente que tecnotipológicamente están relacionadas con las que aparecen en los niveles VIII a VI de la secuencia solutrense de La Lluera I (Rodríguez Asensio 1990, 1992).

En lo referido a material soporte se documenta dentro de la serie industrial una ligera preponderancia de la cuarcita $(53,33 \%)$ frente al silex $(46,67 \%)$, circunstancia que viene explicada por los tipos que componen la muestra: puntas de cara plana y raederas principalmente para el caso de las cuarcitas y raspadores, perforadores y piezas de retoque continuo, de pequeño formato, en el sílex. Inscribiéndose esta circunstancia en las normas industriales descritas para el Solutrense cantábrico (Rasilla 1994) y que también se encuentran presentes en la serie industrial solutrense de La Lluera I.

Esta paridad industrial se ve profundamente alterada al analizar el registro de los productos de talla documentados donde cabría esperar, como ocurre en La Lluera I, una mayor presencia del sílex respecto a la cuarcita, circunstancia explicada por las labores de talla. En el caso de La Lluera II se registran sólo 9 lascas y 1 hoja en sílex frente a 21 lascas y 7 hojas en cuarcita, $26,32 \%$ y $73,68 \%$ respectivamente, todas ellas de pequeño formato en el caso del sílex y de mediano y gran formato en el de la cuarcita. Dándose la circunstancia añadida de no apreciarse remontajes entre ellas y presentar en todos los casos tanto las aristas como los bordes frescos y cortantes.

Llegados a este punto y con la necesaria prudencia entendemos, por las razones expuestas, que esta pequeña colección industrial ha de tener un origen externo a La Lluera II y que únicamente fue depositada o abandonada en ella. Viene a apoyar esta opinión el hecho de que las 7 hojas de cuarcita presentes, de gran formato, son coincidentes en todos los aspectos con un grupo de hojas documentadas únicamente en el registro industrial del estrato $\mathrm{VI}$ de La Lluera I y aunque no se aprecian remontajes entre los ejemplares de ambas cuevas, todo parece indicar que todas pertenecen de salida a un mismo núcleo. Esto induce a pensar que la colección presente en La Lluera II tiene su origen y elaboración en La Lluera I y más concretamente en el momento de ocupación documentado en su estrato $\mathrm{VI}$, adscrito cronológicamente a la fase 3 o final del Cantábrico I (Hoyos 1994, 1995). 
Lo expuesto también induce a pensar que este registro industrial ha de estar necesariamente relacionado de algún modo con las representaciones artísticas que se desarrollan en la pared occidental. Relación que no puede ser atribuida a las labores propias de ejecución de los grabados, ya que ni se documentan los tipos característicos descritos para ello, ni los elementos líticos presentes en la colección conservan trazas de haber sido utilizados para éste $u$ otro menester en modo alguno.

\section{LAS MANIFESTACIONES ARTÍSTICAS}

En cuanto a las manifestaciones artísticas (Fig. 6), que fueron objeto de un estudio preliminar durante los trabajos de excavación de la cueva (Fortea 1989, 1990), se encuentran agrupadas en un único panel directamente iluminado por la luz solar donde, mediante la técnica de grabado de trazo simple y con ejecuciones tanto superficiales como profundas, así como con algunos rebajes volumétricos de la pared soporte, se desarrolla una serie iconográfica de signos triangulares de diferentes tamaños y disposición en clara asociación con, al menos, una figura de cierva estilísticamente idéntica a las que se encuentran profusamente representadas en los paneles de La Lluera I (Fortea 1994, 2001, 2005).

El carácter del signo triangular en esta composición de La Lluera II obedece en todos los casos a un patrón de eje- cución idéntico, se trata de triángulos prácticamente equiláteros con la base localizada en la parte superior del signo, en lo que se ha definido como de clara simbología genital femenina, esto es triángulos púbicos; desarrollándose en este panel de un modo exhaustivo y en múltiples variantes de tamaño y disposición espacial entre ellos. En unos casos como elementos aparentemente independientes, en otros conformando series de triángulos que comparten un único trazo como base superior, trazo que a su vez es base de un triángulo que los inscribe a todos o triángulos inscritos unos dentro de otros simétricamente, etc.

La figura de la cierva goza en esta monografía de signos de un papel preponderante en el conjunto de la composición, pues no sólo presenta una posición centrada y relevante en el panel, sino que esta disposición se ve remarcada al estar inscrita en el espacio central dejado por tres triángulos que aparecen unidos por sus vértices. Evidentemente, la correlación de la silueta de la cierva como elemento figurativo común en ambas cuevas marca su íntima conexión cultural y cultual; pero sin embargo, el carácter profundamente monotemático y único de las representaciones triangulares en La Lluera II, asi como lo reducido de sus dimensiones, implica que ésta fue entendida y utilizada por los solutrenses como lugar o santuario anexo de La Lluera I, complemento del santuario principal, y por tanto habilitado para el desarrollo de actividades claramente diferenciadas y necesariamente más íntimas o reservadas.

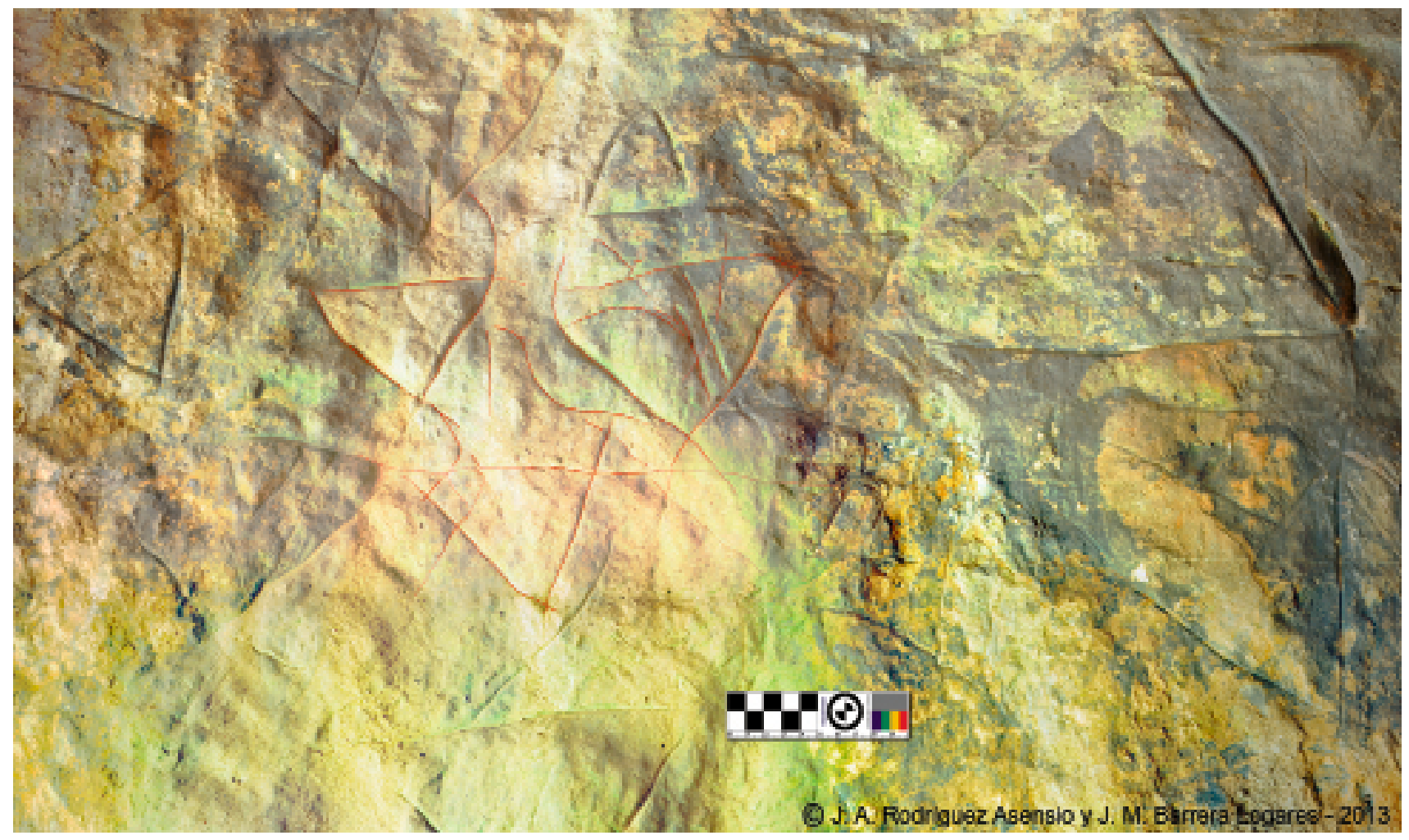

ム Figura 6. Detalle de la zona central del Panel de los Triángulos de La Lluera II. Se destaca con línea roja el grupo de tres triángulos donde se inscribe la figura de la cierva, que marca la íntima relación existente entre este panel y los presentes en La Lluera I. 


\section{CONCLUSIONES}

Sobre una base de conglomerado, conformado por el aterrazamiento del río Nalón de cota $+5 \mathrm{~m}$ y datable en los momentos finales del Laugerie, se verifica un único suelo de ocupación antrópica que, posteriormente, quedará sellado por aportaciones de escorrentía. Este momento de ocupación aporta una industria lítica limitada a unos pocos útiles atribuibles al momento Solutrense en general, sin definir ninguna etapa concreta del mismo, pero que han de ser incluidos necesariamente durante el período Laugerie-Lascaux (Cantábrico I de M. Hoyos). Este único suelo de ocupación es, por ello, contemporáneo a las manifestaciones artísticas que aparecen en una de las paredes del covacho, consistentes en grabados monotemáticos de figuras triangulares entre las que destaca, enmarcada por algunas de ellas, la figura de una cierva de idéntica factura a las documentadas en La Lluera I.
¿Cómo podemos relacionar este covacho con La Lluera I a través de los aspectos geomorfológicos, sedimentológicos, artísticos y arqueológicos? (Fig. 7).

La primera relación, geomorfológica, viene dada intrínsecamente por su génesis paralela. Circunstancia compartida con numerosas oquedades presentes en ambas orillas de esta zona del río Nalón. A saber, pequeñas cuevas y sumideros formados a partir de la disolución de los carbonatos cálcicos por fenómenos de las aguas de filtración y escorrentía provenientes de las vertientes de las laderas circundantes. A estos mecanismos de desagüe vienen a sumarse los producidos por el progresivo encajamiento de la red fluvial, constatados a partir de la terraza de $+8 \mathrm{~m}$ y verificados desde los últimos momentos del Würm III, que mantienen estos desagües kársticos a nivel del río hasta su definitivo destaponamiento en los momentos finales del Würm III-IV, con el desmantelamiento de la terraza $+5 \mathrm{~m}$

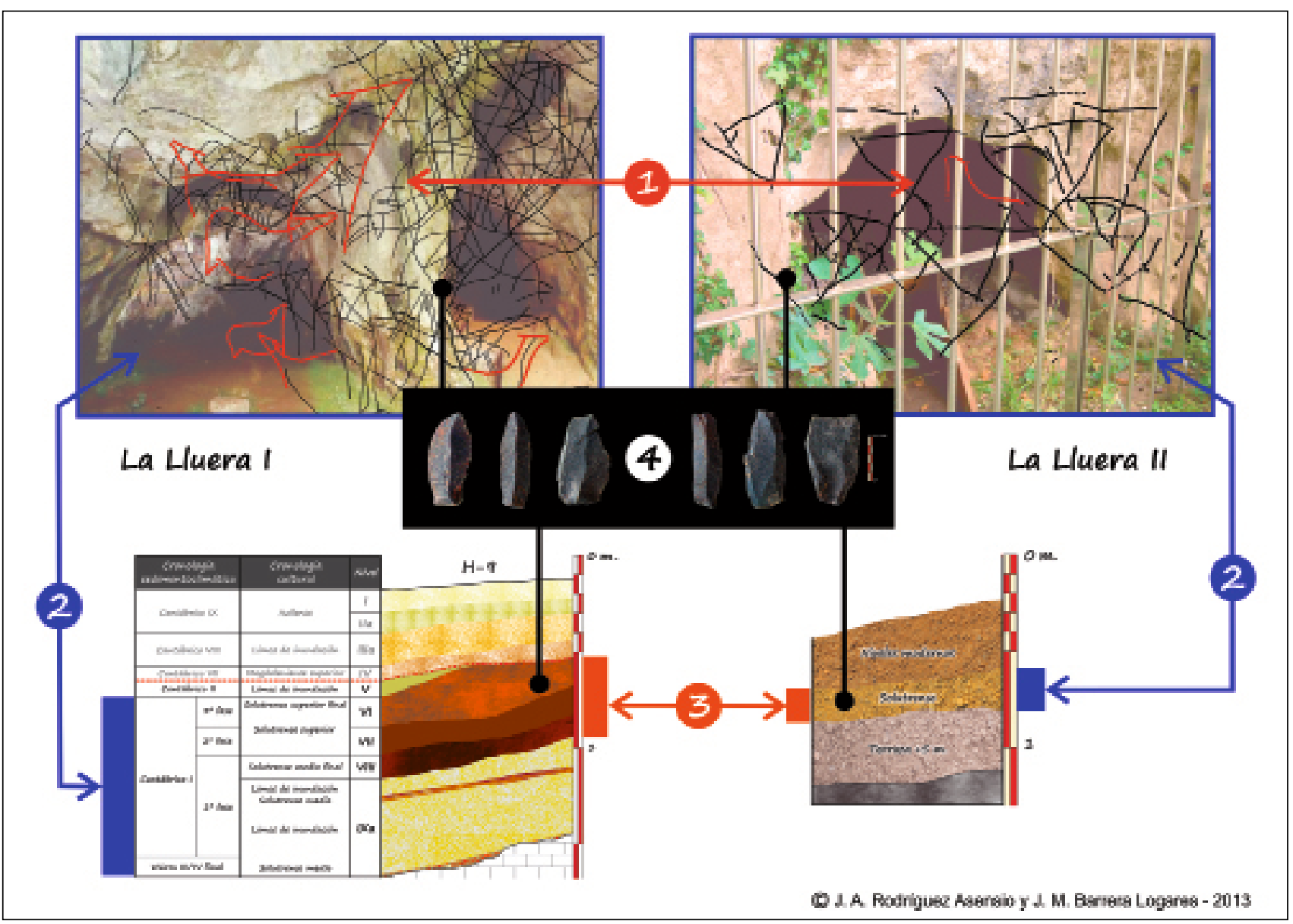

$\Delta$ FiguRa 7. Esquema de las relaciones artístico-arqueológicas de las cuevas de La Lluera I y II: 1, si bien la relación artística entre ambas cuevas puede ser establecida desde sus idénticos parámetros tecnoestlilísticos, destaca como indicador de la contemporaneidad de las manifestaciones gráficas la presencia del motivo iconográfico de la cierva; 2, Esta relación artística se encuentra asociada claramente al momento cultural Solutrense; en el caso de La Lluera I a un arco que queda comprendido entre los niveles IXa VI, y en el caso de La Lluera II al único nivel fértil registrado; 3, Atendiendo a los tipos industriales el paralelismo se verifica en los niveles VIII a VI de La Lluera I, estratos donde se documenta la aparición de puntas de cara plana, elemento que también aparece registrado en el nivel fértil de La Lluera II; 4, Un último elemento de relación lo constituye la documentación en La Lluera II de un grupo de hojas de cuarcita de gran formato que coinciden tecnotipológicamente y materialmente con otro grupo idéntico registrado únicamente en el nivel VI de La Lluera I, lo que implica la contemporaneidad de ambos conjuntos, de los niveles en que se encuentran $\mathrm{y}$, por extensión, de los grabados. 
en el caso de La Lluera I y ligeramente anterior en el de La Lluera II (Hoyos 1994, 1995).

Aunque este hecho no implica que necesariamente ya fueran operativas en esas épocas de cara al asentamiento humano, dada su proximidad al río, y que con las debidas matizaciones y prudencia retrotraigamos esta circunstancia como muy pronto a las pulsaciones iniciales del Cantábrico $\mathrm{I}$, en que el progresivo rigor climático va regularizando y minimizando paulatinamente los procesos de inundación fluvial. A estos momentos, posteriores al desmantelamiento de la terraza $+5 \mathrm{~m}$, atribuimos pues los primeros y escasos restos antrópicos registrados en el nivel IXa de La Lluera I, adscritos por nosotros por afinidad tecno-estratigráfica con la cercana cueva de Las Caldas (Corchón 1981, 1990) como pertenecientes al periodo Solutrense medio. Sedimentológicamente, la relación de ambas cavidades se centra en la presencia de estos restos de terraza, terminus ante quam, base de la serie estratigráfica registrada en las dos cuevas; pues a partir de ella la historia geológica de ambas cuevas de La Lluera será básicamente distinta. Mientras que La Lluera I continuará viéndose afectada por la mecánica fluvial con sucesivos procesos de inundación y vaciado (Rodríguez Asensio 1990), La Lluera II gracias a su ubicación en una cota ligeramente superior únicamente sufrirá las alteraciones producidas por los fenómenos de escorrentía y, evidentemente, por las manifestaciones de la acción antrópica.

La relación industrial entre ambas cuevas viene dada, en principio, por la presencia del tipo de las puntas de cara plana, lo que enmarcaría el suelo de ocupación registrado en La Lluera II en alguno de los momentos documentados entre los estratos VIII a VI de La Lluera I, esto es durante el momento Solutrense superior en general. Sin embargo, la presencia en la colección de un grupo de hojas en cuarcita de idénticas características a otro grupo localizado exclusivamente en el segundo suelo de ocupación del estrato $\mathrm{VI}$, hace que la correlación de las colecciones sea más precisa, pudiéndose ubicar ambas durante la tercera fase del Cantábrico I (Hoyos 1994, 1995). Coincidiendo además esta circunstancia con lo que se ha definido como el momento de ocupación máxima para el período Solutrense de La Lluera I y el momento más verosímil para la realización de los paneles de esta cueva.

La relación artística queda claramente definida técnica, estilística e iconográficamente por medio de la figura de la cierva como elemento común, configurándose de este modo los paneles de ambas cuevas como un todo indisociable. La diferencia vendria dada por el diferente desarrollo iconográfico, que en el caso de La Lluera II se manifiesta en el monotema de los triángulos púbicos. Esta circunstancia, así como las reducidas dimensiones que ofrece el covacho que hacen imposible su habitabilidad indicarian para él un uso cultural más puntual y evidentemente complementario al que los grupos del Solutrense superior del valle del Nalón desarrollaban en el santuario de La Lluera I, por lo que ambas cuevas han de contemplarse en este aspecto como formando parte de un todo, único e indivisible.

\section{BIBLIOGRAFÍA}

Corchón Rodriguez, M. S. 1981: Cueva de Las Caldas. San Juan de Priorio (Oviedo). Excavaciones Arqueológicas en España 115. Ministerio de Cultura. Madrid.

- 1990: "La Cueva de Las Caldas (Priorio, Oviedo). Investigaciones efectuadas entre 1980 y 1986". Excavaciones Arqueológicas en Asturias 1983-1986. Servicio de Publicaciones del Principado de Asturias. Oviedo: 37-53.

Fortea Pérez, F. J. 1989: "Cuevas de La Lluera. Avance al estudio de sus artes parietales". En M. R. González Morales (coord.): Cien años después de Sautuola: Estudios en homenaje a Marcelino Sanz de Sautuola en el Centenario de su muerte. Diputación Regional de Cantabria. Santander: 187-202.

- 1990: "Cuevas de La Lluera. Informe sobre los trabajos referentes a sus artes parietales". Excavaciones Arqueológicas en Asturias 1983-1986. Servicio de Publicaciones del Principado de Asturias. Oviedo: 19-28.

- 1994: "Los "santuarios" exteriores en el Paleolítico cantábrico". Complutum 5: 203-220.

- 2001: "Los comienzos del Arte paleolítico en Asturias: Aportaciones desde una arqueología contextual no postestilistica". Zephyrvs 5354: 177-216.

- 2005: "Iberian palaeolithic rock art". Coalition 10: 8-14.

González Fernández, B.; Menéndez Casares, E.; Jiménez Sánchez, M. y MarTOS DE LA TORRE, E. 2006: "Propuesta de punto de interés geológico: El karst y los meandros del río Nora entre Cayés y Priañes (Asturias)". Trabajos de Geología 26: 149-158.

Hoyos, M. 1989: "La Cornisa Cantábrica". En A. Pérez-González, A., P. Cabra y A. Martín-Serrano, A. (eds.): Mapa del Cuaternario de España. ITGE. Madrid: 105-119.

- 1994: "Caracteristicas Sedimentokársticas de los Interestadios Laugerie y Lascaux en la Cornisa Cantábrica". Férvedes 1: 21-37.

- 1995: "Paleoclimatología del Tardiglaciar en la Cornisa Cantábrica basada en los resultados sedimentológicos de los yacimientos arqueológicos kársticos". En A. Moure Romanillo y C. González Sainz (coords.): El final del paleolítico cantábrico: transformaciones ambientales y culturales durante el Tardiglacial y comienzos del Holoceno en la Región Cantábrica. Servicio de Publicaciones de la Universidad de Cantabria. Santander: 77-117.

RASILLA VIVES, M. DE LA 1994: "El Solutrense de la Cornisa Cantábrica". Férvedes 1: 69-87.

Rodríguez Asensio, J. A. 1990: "Excavaciones arqueológicas realizadas en la cueva de La Lluera (San Juan de Priorio. Oviedo)". Excavaciones Arqueológicas en Asturias 1983-1986. Servicio de Publicaciones del Principado de Asturias. Oviedo: 15-17.

- 1992: "Excavaciones arqueológicas en la cueva de La Lluera II (San Juan de Priorio. Oviedo)". Excavaciones Arqueológicas en Asturias, 1987-1990. Servicio de Publicaciones del Principado de Asturias. Oviedo: 29-32. 
